\title{
La co-création pour un apprentissage des émotions par le biais du livre artistique
}

\section{Lila Merle}

\section{(2) OpenEdition \\ Journals}

Electronic version

URL: https://journals.openedition.org/trema/6531

DOI: 10.4000/trema.6531

ISSN: 2107-0997

Publisher

Faculté d'Éducation de l'université de Montpellier

Electronic reference

Lila Merle, "La co-création pour un apprentissage des émotions par le biais du livre artistique", Tréma

[Online], 55 | 2021, Online since 28 October 2021, connection on 13 December 2021. URL: http://

journals.openedition.org/trema/6531 ; DOI: https://doi.org/10.4000/trema.6531

This text was automatically generated on 13 December 2021.

Trema 


\title{
La co-création pour un apprentissage des émotions par le biais du livre artistique
}

\author{
Lila Merle
}

\section{Introduction}

Des enjeux en termes d'éducation chez le jeune enfant sont présents en dehors de l'école, et visent une formation culturelle. Souvent sollicités, des intervenants, ici des artistes à part entière, jouent un rôle fondamental dans l'éducation des enfants, hors l'école mais aussi au sein même de cette dernière. Cet article met en lumière le lien existant entre le livre artistique et l'éducation, et souligne le rôle d'une pratique artistique spécifique et de ses acteurs pour un apprentissage des émotions chez le jeune enfant. La philosophie de l'éducation permet de se saisir de ce qui se joue dans ces pratiques artistiques en termes d'apprentissages, ici il s'agira de celui, sensible, des émotions. Il s'agit de s'interroger sur une pratique éducative dont le fondement tient à un phénomène de co-création entre un artiste, un enfant et parfois un enseignant. S'agit-il d'une forme d'expérience esthétique du beau (Kerlan, 2004 ; Blomgren, 2019) ? Peut-on y voir une pratique autonome des jeunes enfants/élèves?

2 L'« expérience » et la «co-créativité » favoriseraient un apprentissage culturel et personnel chez le jeune enfant. L'hypothèse serait de voir dans cette conjonction une conception de l'éducation qui ne se limiterait pas à la formation intellectuelle du jeune citoyen. En effet, on peut y voir également une volonté d'offrir une formation culturelle, sensible et émotionnelle. Le support livre peut être une forme d'expression artistique qui tend vers la démocratisation de l'art (Beguery, 2002). Le livre artistique est alors pensé en termes d'espace de socialisation, de rencontre avec l'art et avec un artiste mais aussi de manière plus générale comme une rencontre avec l'altérité. L'école «n'est pas la seule instance de socialisation » (Dewey, 1916). 
3 Cet apprentissage vise à la préparation de l'enfant pour lui permettre de devenir un acteur éveillé et éclairé de la société (Nussbaum, 2011). C'est par l'expérience esthétique des Arts (Kerlan, 2005 ; Chirouter, 2015), par la coopération et les échanges que les temps des ateliers artistiques peuvent permettre un apprentissage des émotions démocratiques (Nussbaum, 2011). Cet apprentissage des émotions suggère le développement de capacités à vivre ensemble, à prendre place au sein d'un groupe, à participer à la construction du commun (Zask, 2007). Lors d'un atelier, c'est en se positionnant et argumentant sur les émotions que l'enfant ressent au contact d'une œuvre qu'il prend place dans l'environnement qui l'entoure; il va également confronter ses émotions à celles des autres enfants. C'est ce que Zask (2007) nomme la rencontre avec soi mais aussi avec le commun, l'expérience de l'altérité, du monde.

\section{Un objet culturel pour un apprentissage des émotions : Le livre artistique ou Artist's Book}

4 Le livre d'artiste est un objet d'art né de l'avant-garde des années 60. «En travaillant avec la banalité du livre, les artistes rompent avec une tradition, la leur, celle des Beaux-Arts, mais pour nouer des liens inédits avec une autre tradition, celle du livre " (Moeglin Delcroix, 2012). Le livre devient ainsi un moyen de diffusion, permettant une plus grande facilité de reproduction, il est aussi une alternative d'exposition et d'expression (Dupeyrat, 2012).

5 Tenter de définir ce qu'est le livre d'artiste pour enfants est, comme l'affirme l'artiste Sophie Curtil « une chose bien hasardeuse ». Le livre d'artiste pour enfants se définit par «ce qu'il n'est pas» (Merle, 2017) et ceci suscite déjà des interrogations. Le livre d'artiste est un livre dont « l'artiste conçoit le texte et l'illustration, il revêt alors la forme d'une œuvre d'art» (Moeglin Delcroix, 2012). Cette définition identifie clairement l'artiste comme le concepteur et le livre comme une œuvre. Il est une œuvre « originale même s'il est reproduit en plusieurs exemplaires, et l'artiste est concepteur du contenu et de la forme " (Moeglin Delcroix, 2012). Le livre d'artiste est synonyme d'art contemporain, d'art conceptuel, minimal, inspiré et initié notamment par le mouvement artistique du Black Mountain College (Zask, 2014) ou l'art est considéré comme un modèle de pratique démocratique. Selon Zask, «il existe dans l'expérience de l'art [...], une dimension politique ». Certes ce n'est pas au sens "politicien» du terme, mais plutôt en lien avec l'idée de rencontre. Cette pensée de la philosophe fait écho à ce que Dewey avait lui-même exploré dans L'art comme expérience (1934). Le contact avec l'autre va venir modeler, modifier voire structurer l'individu. Dewey aimait à penser que c'est par l'association, le collectif, le contact avec l'autre que le développement de soi est rendu possible, et que le processus d'individuation peut ainsi entrer en jeu. Ce processus permet au sujet, en coopérant avec autrui, de devenir luimême en tant qu'individu. Zask propose de prolonger la pensée de John Dewey en précisant que la participation démocratique relève d'une rencontre. Ce serait en prenant part aux débats, aux expériences de rencontres que l'on apprendrait la citoyenneté, que l'on apprendrait à être acteur et actif dans notre environnement. Le commun apparait comme l'aboutissement d'un long processus au cours duquel les personnes concernées se font entendre, modèlent leur point de vue d'après la manière dont les autres expriment le leur, délibèrent. 
6 Enfin le livre d'artiste est un espace de rencontres entre un artiste et son lecteur, un objet singulier, un livre insolite, un ouvrage rare et précieux (Brogowski, 2010). On peut également dire qu'il est une œuvre d'art hybride, en référence à l'hybridité des pratiques et formes dans le champ artistique, considérant ainsi le livre d'artiste comme un croisement de l'art et de la littérature, comme le lieu d'une expérience de création.

7 À la recherche du beau dans le livre pour enfant, les auteurs proposent des expériences littéraires, esthétiques et artistiques grâce aux choix de certains éditeurs. Dans le milieu éditorial on interroge l'enfance, ce qui questionne du même coup les experts comme Jocelyne Béguery (2002) : «La rencontre d'œuvres et de gestes artistiques avec le monde de l'enfance témoigne-t-elle pour notre époque d'une pensée de l'art et d'une pensée de l'enfance?». Certaines maisons d'éditions ont placé l'enfant au centre de la recherche artistique et littéraire. Elles ont fait le choix de techniques plastiques en donnant la primauté à l'image sur le texte ; l'image constituant à elle seule la trame narrative. Ces maisons d'éditions sont tournées vers une recherche esthétique.

Le livre d'artiste ou livre artistique fait partie de ces objets de l'environnement enfantin qui alimente la réflexion en philosophie de l'éducation en ce qu'il renseigne sur l'évolution du sentiment de l'enfance (Brougère, 1995). Par ailleurs, on observe que l'année 1968 tourne définitivement la page avec les modèles traditionnels que l'on pouvait trouver dans les livres pour enfants. Les artistes comme Paul Cox ou encore Louise-Marie Cumont, Katsumi Komagata vont continuer le mouvement que des pionniers issus de l'avant-garde russe comme Nathalie Parain, ou encore l'américain Rémy Charlip, et aussi le designer italien Bruno Munari ont initié en repoussant de plus en plus les limites entre le monde dit enfantin et celui de l'adulte. À tel point que certains éditeurs comme Delpire ne verront plus l'enfant en tant que destinataire spécifique, mais plutôt comme un alter ego des grandes personnes. Il n'y a pas de différence entre les structures de l'imaginaire enfantin et celles de l'imaginaire de l'adulte. Au même titre que l'album pour enfants, les livres artistiques peuvent rejoindre l'histoire de la littérature d'enfance et de jeunesse. On peut donc prendre pour témoins les livres artistiques pour tenter de construire une histoire de l'enfance, témoins également d'une nouvelle forme d'éducation: éducation par les Arts, éducation par une pratique artistique spécifique. Ci-après, quelques livres artistiques utilisés auprès des enfants :

9 -Charlip, R. (2000). Its Look Like Snow- On dirait qu'il neige. Les Trois Ourses.

-Charlip, R. (2011). Reading Dance. Édition Minimondi.

-Cumont, L-M. (2000, 2009). Au lit! Livre en tissu réalisé à la main, signé et numéroté.

Édition MéMo.

-Cumont, L-M. (1994, 2009). Les chaises, livre en tissu réalisé à la main, signé et numéroté. Édition MéMo.

-Curtil, S. (2001). Ali ou Léo ? Co-édition Les Doigts Qui Rêvent/Les Trois Ourses.

-Hoban, T. (1971, 2003). Look Again / Que vois-tu ? MacMillan. Édition Kaléidoscope.

-Komagata, K. (2008). Little Tree. Édition bilingue japonais-anglais, One Stroke.

-Komagata, K. (1990). Meet Colors. Kasei-sha.

-Komagata, K. (1990). Walk and look. Kasei-sha.

-Lavater, W. (1965). Le petit chaperon rouge. Édition Maeght.

-Munari, B. (1980). Les Prélivres. Danese, Milan. Édition Corraini, 2002.

-Munari, B. (1999). Dans la nuit noire. Édition Seuil Jeunesse.

-Parrain, N. (2006). Mon chat. Édition MéMo, la collection des Trois Ourses. 
10 A l'examen de cette sélection d'ouvrages, on constate qu'il y a peu ou pas de texte dans ces livres. Soit il est présent sous la forme d'une phrase (komagata, 2008), d'une légende (Lavater, 1965). La graphie est singulière et fait appel aux compétences de l'artiste, à ses matériaux de prédilection: peinture, tissus, photographies, papiers. Ce sont les matériaux qui vont retranscrire les mots et permettre à l'enfant de s'approprier le livre quand bien même il ne sait pas encore lire. L'obstacle de l'écriture, et nous pouvons aller plus loin en parlant de l'obstacle de la langue sont gommés par l'usage du langage plastique.

11 Si le langage utilisé n'est pas l'écriture, que l'on retrouve dans un livre, la structure du texte est aussi modifiée. Il n'y a pas de trame narrative rigide, pas plus que de schéma quinaire que les enfants ont coutume de rencontrer dans les albums, la trame est plutôt intuitive: ce qui permet à l'enfant de l'inventer, de se l'approprier en parfaite autonomie. Il y a peu ou pas de personnage. Les personnages étant souvent représentés par des formes, des couleurs (Warja Lavater, 1965). Le format du livre sert également de structure.

Comparable à un squelette, dont la colonne vertébrale serait la reliure, le livre se compose comme un tableau, où chaque page, en place du texte, propose une structure plastique.

En retirant le texte, le langage visuel et l'artiste jouent avec l'enfant et lui proposent ce que nous nommons une authentique expérience sensorielle. L'enfant regarde, manipule, touche, expérimente avec tous ses sens et va apprendre à regarder, voir, chaque détail, expérimenter les matériaux avec tout son corps. Pour certains livres d'artiste on peut même parler du corps qui devient narrateur. Au sens où l'artiste, par le jeu des formes, des couleurs, des matières et matériaux va solliciter la participation du jeune lecteur. Ce système permet à l'enfant de rentrer véritablement dans le monde de l'artiste et de rencontrer l'univers de ce dernier. L'expérience sensible, sensorielle et esthétique vont bouleverser l'acte même de lire. L'enfant pourra en effet lire, ou du moins raconter l'histoire à l'adulte. Le livre d'artiste, au-delà de l'imaginaire qu'il suggère, invite aussi à créer, à prendre part au processus créatif avec un artiste dans un espace-temps singulier que représente l'atelier artistique.

«Être créatif c'est avoir la capacité d'imaginer et de donner forme à des idées, des choses, c'est trouver des solutions inédites, originales, efficaces à des problèmes " (Louise-Marie Cumont, 2016).

Le livre d'artiste propose à l'enfant d'expérimenter le processus créatif. Il est considéré comme un espace de jeu et de création qui offre une multitude de possibilités tant pour l'artiste que pour l'enfant. Il est un lieu de re-création, une expérience du monde (Chirouter, 2015 ; Merle, 2017). Comme ce livre contient rarement de texte, ou peu - la trame est laissée volontairement souple - il donne simplement une direction, que l'enfant est libre de choisir ou interpréter. Ceci lui permet de créer, de donner libre cours à son imaginaire, L'œuvre d'art est en lien avec les expériences du regardeur mais aussi avec notre imaginaire (Ricoeur, 1983). Dans certains livres d'artiste le corps du regardeur peut devenir narrateur au sens où l'artiste, par le jeu des formes, des couleurs, des matières et matériaux va solliciter la participation active du jeune lecteur. 


\section{Le dispositif mis en œuvre}

15 Le dispositif a été mis en place au sein de deux classes de maternelle sur une période de deux années scolaires. Le dispositif fut expérimenté auprès d'enfants de deux niveaux de classe différents : la moyenne section (4 ans) et la grande section ( 5 ans). Soit 56 enfants au total. Un protocole fut respecté et conservé à l'identique sur les deux années et dans les deux classes :

16 - une lecture découverte offerte d'un livre artistique par l'enseignant.e en grand groupe - un workshop auprès d'un artiste en petits groupes

- un débat - retour sur l'expérience vécue en grand groupe

- un accès libre à l'œuvre au coin bibliothèque

18 - leur curiosité, mesurée par leurs questions ou réactions sur l'espace et le temps du workshop,

- leur concentration, mesurée par le temps passé dans l'activité par l'enfant,

- leur rapport aux autres, les interactions entre pairs,

- la construction de leur argumentation, leur langage au moyen d'une analyse du discours

-leur rapport au monde à travers notamment l'analyse de l'utilisation des pronoms personnels.

19 L'école où ont été mis en place les ateliers est située en proche banlieue lyonnaise. Elle regroupe des enfants de langues maternelles différentes. Les séances d'une durée moyenne de 30 minutes ont été enregistrées en version audio et vidéo puis analysées à partir de la retranscription mais également et surtout au moyen des dessins réalisés par les enfants.

20 Le livre d'artiste modifie les lieux de l'art puisqu'il permet de se vivre en dehors des musées ou galeries d'art. Les ateliers et les lectures collectives des livres d'artistes participent au déplacement de l'art au sens où l'art ne se limite plus aux institutions culturelles mais bien ex situ où l'art se produit dans un espace inattendu. À la manière des performances ou happenings du mouvement Fluxus des années 60, l'art est partout. Il est dans la rue, à l'école, mais surtout hors de l'école, comme par exemple à la bibliothèque. L'idée est de permettre à chacun d'expérimenter l'art avec ce qu'il est. Le spectateur, le jeune lecteur devient co-créateur d'une œuvre. Le duo, composé du livre d'artiste et de l'atelier, marque cette volonté de proposer à l'enfant un art accessible et dans un même temps, il est révélateur d'une autre définition de l'art et de l'enfance. Le livre devient un lieu de rencontres et d'échanges où chacun va pouvoir s'exprimer, apprendre mutuellement des uns et des autres. Le livre et l'atelier créent un nouvel espace d'expression, de création et d'expérience sensorielle.

21 La liberté des enfants s'exprime aussi dans le fait que les artistes gardent le cap fixé par les enfants pendant les ateliers. Ce sont eux qui orientent les actions, les événements lors de l'atelier. Rien n'est imposé à l'exception des matériaux. Il n'y a pas « d'attendus » de résultat comme à l'école, ici, ce qui intéresse l'artiste c'est le parcours, le cheminement emprunté par l'enfant pour résoudre son propre problème plastique. L'artiste guette « l'inattendu », la surprise, l'imprévu de ce que le groupe d'enfants va produire. Il ne prévoit rien du « produit fini » si ce n'est son matériel. Cette surprise va émaner du groupe et de la création commune ; elle est permise par le libre-échange, la 
communication entre les enfants, avec l'artiste, qui fait de l'atelier un lieu de rencontre privilégié avec l'art et l'univers d'un artiste, des pairs et de soi-même.

L'expérience esthétique est au sens premier l'expérience du beau. Le beau ne s'entend pas ici au sens commun - c'est-à-dire le qualificatif de quelque chose de joli, agréable à regarder - mais bien au sens philosophique comme un processus créateur de sens et un nouveau moyen d'accéder à la connaissance par les sens et l'expérience sensorielle. L'espace créé par le livre artistique, la Beauty Bubble (Blomgren, 2019), est formé d'un objet, d'un sujet et d'un environnement. L'importance de l'espace est mis en lumière en précisant que:

«Philosophical aesthetics, with its sensitive cognition, marks aesthetic processes as creative processes alternating between the subject and the world and such processes changing the people and surroundings involved» $(2019$, p.5).

On comprend donc que la perception et représentation de cet environnement vont également jouer un rôle, venir modifier le processus créatif et de fait l'expérience esthétique. La Beauty Bubble (Blomgren, 2019) constitue un environnement propice à l'appropriation, l'expérience et l'action du « regardeur ». Ce dernier va pouvoir agir sur l'œuvre. L'œuvre d'art est innovante dans ce qu'elle suggère sur l'instant (Eco, 1965), et créatrice dans ce qu'elle autorise : un autre espace-temps, une projection d'un monde, le quasi-monde (Ricoeur, 1983). La perception d'une œuvre serait le résultat d'un entrelacement de la réception et de la spontanéité, où la réceptivité revêt une dimension passive et sensorielle; quant à la spontanéité, elle tend vers une dimension active où l'individu va " pouvoir et agir ", où il pourra se dire : « je peux et/ou je fais ». L'espace créé issu de la rencontre entre le spect-acteur (Ruby, 2002) et l'œuvre n'est donc pas vide. Il peut être au contraire compris comme un espace de permission, d'autorisation pour vivre une expérience artistique, esthétique.

D'autres conditions vont permettre à l'œuvre de révéler tout son potentiel et ses possibilités en termes d'apprentissage des émotions comme par exemple l'expérience du commun (Zask, 2007) au sens de communauté : «Le commun apparait comme l'aboutissement d'un long processus au cours duquel les personnes concernées se font entendre, modèlent leur point de vue d'après la manière dont les autres expriment le leur, délibèrent et ajustent leurs attentes mutuelles » (Zask, 2007).

Une autre condition permet à l'expérience esthétique de se produire : La poétique de l'œuvre (Umberto Eco, 1962). En effet, l'œuvre d'art délivre un message composé de signifiés (symboles) qui forment un seul signifiant (l'œuvre). Cette condition va permettre à l'enfant de se constituer un répertoire de signes et symboles lui permettant de s'approprier un univers artistique, de participer de manière active et consciente au processus créatif. L'appropriation peut se décliner sous trois formes: $1 /$ comprendre pour créer, 2/ lorsque l'esprit s'approprie pour faire siennes les connaissances qu'il acquiert, 3 / l'appropriation comme une forme émancipatrice et stimulante.

La perception d'une œuvre va dépendre de nos expériences personnelles, valeurs, et, plus largement, de processus de significations (Dewey, 1922). Chacun va donc percevoir, ressentir ce qui lui est propre en fonction de son expérience, de son vécu. Ce que chacun perçoit est indissociable de son contexte, de son histoire sociale et physique (Aden, 2019). L'expérience esthétique est un « tout » formé par la somme de toutes ces variables. 


\section{Vers un apprentissage des émotions démocratiques pour devenir un citoyen éclairé et acteur de la cité}

Nussbaum (2011) fait état d'une crise qui traverse le monde. Il ne s'agirait pas d'une crise économique mais plutôt d'une crise qui traverse le système éducatif, et qui touche la formation des futurs citoyens de la démocratie. Elle fait état d'une dégradation des relations entre les citoyens qui ne parviennent plus à vivre ensemble ou trouver de consensus pour le commun.

Pour pérenniser la démocratie, un apprentissage des " émotions démocratiques » serait un moyen d'anticiper cette crise car c'est en choisissant une éducation pour la démocratie que les citoyens pourront participer activement et consciemment aux décisions de la cité. L'apprentissage des émotions et notamment celle de l'empathie serait une possibilité tournée vers une éducation moins intéressée par le profit ou la compétition qui peuvent parfois engendrer des comportements violents. Le but étant de proposer un modèle éducatif plus respectueux des valeurs d'une société démocratique, des autres et de soi, les émotions démocratiques peuvent être acquises lorsque des conditions sont réunies. Notamment par la rencontre avec l'autre, l'expérience de l'autre et la conscience d'être-au-monde. Le développement de l'empathie chez le sujet ne va pas de soi. Pour cela il convient de l'apprendre dès le plus jeune âge par une forme singulière d'enseignement.

Trois principes et conditions seraient favorables pour un apprentissage des émotions démocratiques :

31 1/ La pratique du débat

La pratique du débat rejoint la pensée et la pratique. C'est une forme de pédagogie dite " socratique ", c'est-à-dire une éducation où la réflexion, l'esprit critique naissent de la pratique du dialogue et des échanges. Et c'est par l'exercice de la pensée critique que l'individu pourrait accéder de manière plus active et consciente à la vie démocratique.

32 2/ Le souci de former des citoyens du monde

Ce principe entre en résonance avec une vision de la société vue comme multiculturelle donc ouverte sur le monde. L'éducation doit former « un membre actif, critique, réflexif et empathique d'une communauté d'égaux» (Nussbaum, 2011, p177). Pour ce faire, l'enfant doit, dès le plus jeune âge, être mis en relation avec l'autre, apprendre à raisonner, argumenter et réfléchir avec autrui pour rechercher le consensus.

33 3/ La promotion de l'imaginaire à travers la littérature et les Arts auprès des jeunes enfants

C'est vers ce troisième principe que tendent l'expérience esthétique des Arts et la promotion de l'imaginaire à travers la littérature et les Arts auprès des jeunes enfants. Le principe énoncé peut être reformulé ainsi : par la coopération et les échanges qu'une pratique des Arts et de la littérature propose, le sentiment d'empathie appelé aussi "émotion démocratique» pourrait se développer. Ceci est possible d'autant plus lorsqu'il s'accompagne d'une médiation de la part des acteurs : enseignants, artistes et enfants. Mais l'œuvre d'art aussi, en elle-même, va jouer un rôle fondamental pour que la médiation soit optimale. Une lecture permet de rendre compte de catégories favorisant la signification d'une œuvre. La référence fait appel à des éléments précis et connus du spectateur. Mais chacun sera libre de se saisir de ce qu'il souhaite par rapport son expérience personnelle et ses connaissances. La suggestion fait appel (ou 
pas) à des éléments plus abstraits et éloignés du sujet notamment l'imaginaire du spectateur (Eco, 1962). L'œuvre est dite ouverte lorsque le spectateur peut agir sur l'œuvre tel que prévu par l'artiste (ce n'est donc pas une co-conception avec l'artiste). L'œuvre est dite achevée (dans sa forme) quand le sens a été fixé par l'artiste et qu'elle est prête à être consommée sans action du spectateur sur l'œuvre. L'œuvre est dite interactive, selon Ricoeur (1974), lorsqu'elle ouverte et achevée, faisant appel au "quasi-monde » c'est-à-dire à la jonction entre le monde du spectateur et celui de l'artiste lorsque l'artiste et le spectateur co-conçoivent l'œuvre.

L'œuvre ouverte serait plus signifiante que l'œuvre achevée car elle favorise la participation active du spectateur dans le processus de création mais le regardeur est libre de participer ou non. L'œuvre est œuvre de manière autonome. L'œuvre ouverte serait plus signifiante que l'œuvre achevée car elle intègre l'expérience et/ou les connaissances du spectateur. L'œuvre interactive serait plus signifiante que les deux autres types d'œuvres car elle permet de concilier les deux dimensions fondamentales d'une œuvre: la fonction référentielle de l'artiste et la fonction de suggestion du spectateur.

La réceptivité et la spontanéité sont rendues possibles par l'imagination convoquée par l'œuvre d'art. L'imagination est la capacité permettant « d'opérer les synthèses faisant voir les ressemblances inattendues et nous permettant d'entrer dans un univers qui révèle la réalité sous un jour nouveau » (Thomasset, 2005). Pour Ricoeur (1983), l'œuvre va permettre au spectateur de développer son imaginaire. Cette imagination développée possède trois grandes fonctions qui vont influer sur la perception, la réception, l'appropriation et les actions du spectateur vis-à-vis de l'œuvre. Ces trois fonctions de l'imagination permettent de modéliser le concept d'appropriation d'une œuvre par le spectateur, et de comprendre quelles peuvent être les raisons et les conditions favorisant la mise en relation signifiante entre une œuvre et le public.

La fonction de schématisation offre un visuel au spectateur que l'artiste donne à regarder, et rend visible, sensible et tangible. De même que dans le récit d'une œuvre littéraire l'usage de métaphores permet de "voir » une idée abstraite, le plasticien va, quant à lui, avoir recours aux matériaux (peinture, argile, pierre, etc.) et à sa technique plastique pour rendre tangible sa démarche artistique. La fonction de schématisation de l'imaginaire se trouve également dans ce qu'elle va permettre, à des sujets/objets $a$ priori asymétriques, de se rapprocher pour créer un sens nouveau, inédit.

37 Une autre fonction de l'imaginaire est celle de retentissement où l'imaginaire $d u$ spectateur va être comme éveillé. Au-delà du regard premier sur l'œuvre, cette fonction de retentissement donnera une dimension plus intime, plus interpersonnelle au travail artistique. En cela, l'imaginaire, par cette fonction, va venir raviver, mettre à nouveau en lumière des expériences antérieures, des souvenirs jusque-là enfouis du spectateur. C'est ce que Umberto Eco (1965) nomme la Poétique de l'œuvre, ou le quasimonde (Ricoeur, 1986). C'est ce kairos où l'artiste, par un jeu d'alternance entre référence et suggestion, va offrir au spectateur la possibilité d'explorer l'œuvre avec son vécu, ses connaissances et son environnement culturel et social.

38 Enfin, la fonction de désengagement est celle qui va activer le processus de rupture et de prise de recul chez le spectateur: une rupture par rapport au réel. En d'autres termes, le spectateur va regarder, percevoir, recevoir l'œuvre de l'artiste, puis son imaginaire en action va lui permettre d'effectuer une rupture avec ce que lui propose le travail artistique. Cette mise à distance avec le réel (l'œuvre proposée par l'artiste) va 
donner lieu chez le spectateur, à des possibilités en termes d'innovations, d'explorations, de créations, de manières d'être-au-monde. Par ce désengagement, le sujet va pouvoir s'autoriser à essayer, expérimenter d'autres voies que celle(s) indiquée(s) par l'artiste. C'est sur ce point que l'on peut observer des différences lors d'ateliers artistiques ou lors d'expositions. Chacun aura alors la possibilité de s'autoriser (ou non) à faire l'expérience d'un dispositif artistique. On peut observer sur ce point précis des résistances de la part de certains spect-acteurs pour qui il sera impossible de se désengager totalement et librement (Merle, 2017).

\section{Résultats et discussion}

\section{Des Différences entre les enfants de moyenne et grande section}

Les indicateurs choisis ont permis de mettre en lumière que les enfants de grande section avaient augmenté leur temps de concentration de manière plus significative que les enfants de moyenne section. Au fil des séances, les enfants de grande section sont passés de 15 minutes à 30 minutes de concentration, contre 20 minutes pour les enfants de moyenne section. Cette différence peut s'expliquer par l'âge mais aussi leur capacité d'apprentissage plus élevée.

Il est une autre différence celle du partage de l'expérience aux parents, la curiosité des enfants. Si les enfants de grande section ont partagé volontiers leur expérience auprès de leur famille, les enfants de moyenne section, eux, ont gardé pour eux leur expérience. Là aussi on peut expliquer ce phénomène par l'âge, mais on peut également dire qu'il reste encore difficile pour les enfants de moyenne section de s'exprimer quant à leur vécu, leur ressenti et ce malgré le dispositif mis en œuvre. On peut donc dire que les effets du dispositif sont à nuancer concernant les enfants de 4 ans. Les enfants de grande section ont fait état de leur expérience et ils prennent plaisir à partager auprès de leur famille leur envie de prolonger l'expérience en formulant des souhaits du type : aller aux musées ou à la médiathèque.

\section{Des points communs entre les enfants de moyenne et grande section}

41 L'utilisation du pronom "je » est observable dans le discours des enfants lors des débats après les ateliers. Au fil des séances, les enfants de moyenne et grande section sont capables de prendre la parole en public, d'élaborer une pensée logique sur ce qu'ils ont vécu sur le temps de l'atelier. Ils identifient de manière plus fluide leur participation, les actions qu'ils ont effectuées et le résultat produit. Le pronom " on » est également présent dans le discours des enfants. Si en début d'expérience les enfants sont dans l'action de façon individuelle, on peut voir une coopération se mettre à l'œuvre au fil des séances. "Je fais, je suis » devient "on va faire» et " on a fait ensemble ». Ceci est observable dans les discours, lorsque l'on fait le bilan de la séance avec les enfants, mais c'est aussi visible dans les productions plastiques à l'issue des ateliers.

Enfin, l'argumentation des enfants de grande section s'affine. En fin d'expérience, ils sont capables de formuler ce qui leur a plu, déplu et ils justifient pourquoi : «J'aime parce que... ». 
Une pratique des Arts semble plus signifiante pour les enfants car elle favorise la participation active du jeune enfant dans le processus de création et le développement de la réflexion. On peut aussi identifier une forme d'appropriation d'un univers artistique. Plus les enfants pratiquent les Arts, plus ils développent/réveillent leur potentiel, s'approprient leurs Capabilities (Nussbaum, 2011). Ainsi ils coopèrent, échangent, recherchent le consensus. On part du potentiel présent pour aller vers le développement de capacités cognitives transversales. L'art ouvre ainsi le terrain des possibles. Le temps de l'atelier est le Kairos pour «s'autoriser à » expérimenter, tâtonner, faire des erreurs.

Plus les enfants pratiquent plus ils développent et expérimentent leurs émotions notamment la bienveillance, le respect de l'Autre et de soi et l'empathie. On voit notamment se développer l'entraide, l'écoute entre pairs des propositions de chaque membre du groupe. L'expérience des Arts et une pratique régulière des Arts favorisent la rencontre avec l'Autre et avec soi. Apprentissage de «l'être-au-monde ».

D'un point de vue philosophique, on peut convoquer la notion du commun pour évoquer le principe de démocratie qui semble le plus fondamental. Il est important de développer l'imaginaire pour être en mesure d'affronter, faire face à des situations concrètes de la vie réelle. L'imaginaire doit se développer pour permettre un ancrage dans la réalité. Pour ce faire, Dewey (1922) précise qu'il faut partir du potentiel de l'enfant - son imaginaire et ses jeux - pour aller au-delà. En raison de son pragmatisme, l'éducation est conçue comme une action de captation des savoirs applicables dans la vie concrète (présente et future). Ces différents savoirs sont immédiatement applicables dans la réalité de l'enfant. L'école doit permettre à l'enfant de résoudre certains problèmes de la vie concrète et sociale dans l'immédiat et l'avenir. La pratique des livres pour enfants et le livre artistique semblent des outils favorables pour répondre aux besoins de l'enfant.

resture, la fiction, vont créer un espace entre le réel et l'imaginaire du lecteur. C'est le quasi-monde (Ricoeur, 1986) : un laboratoire d'expérimentation de l'esthétique et du sensible entre le réel et l'imaginaire. L'importance de la littérature dans ce qu'elle propose comme outils pour permettre, à partir de la fiction, de se construire un répertoire de symboles, des outils nécessaires pour comprendre et appréhender le monde réel est donc observé.

En quoi ces médiations culturelles singulières autour du livre et les ateliers artistiques peuvent-elles contribuer aux apprentissages scolaires?

Un premier argument avancé est celui de diminuer les inégalités scolaires et permettre à chacun d'accéder à la culture. La multiplication des classes à projet artistique et culturel, les modifications successives des programmes scolaires pour faire une place plus «noble» aux Arts, l'augmentation du nombre de résidences d'artistes dans les écoles, les recherches portant sur les effets de l'éducation artistique et culturelle sont autant de signes qui font ressortir une forme de volonté profonde de démocratiser les Arts (Bordeaux et Kerlan, 2016). Mais pas uniquement. Des objectifs et des effets de ces pratiques sont attendus sur les apprentissages des élèves. En somme les Arts sont censés permettre davantage là où d'autres disciplines ne réussissent pas. S'il est vrai que les Arts peuvent être un terrain et terreau des possibles favorables au développement des émotions, de compétences et capacités nécessaires aux apprentissages, on ne peut cependant pas nier l'apport intellectuel des autres champs d'enseignements. 
futurs citoyens pour leur permettre d'être pleinement conscients de leur environnement mais aussi de leur rôle à jouer dans ce même environnement. Les Arts sont un recours dans des pratiques éducatives car ils sont des outils accessibles aux élèves leur permettant de vivre l'expérience émotionnelle de l'autre mais aussi de soi. L'œuvre d'art s'efface alors comme médium pour laisser libre cours à un rapport imaginaire/imaginant, rapport à l'Autre, où le sujet est créateur, sans cesse saisi dans un arrêt qui l'engage à penser plus loin. La création qui pourrait être considérée comme un espace- temps éphémère, prend une tout autre temporalité dans ce qu'elle autorise d'imprévisible saisissable dans la continuité de la durée. «La nouveauté constitue la trame du réel [...] La réalisation apporte avec elle un imprévisible rien qui change tout » (Bergson, 2009).

\section{BIBLIOGRAPHY}

Beguery, J. (2003). Entre voir et dire. Images de l'art à l'adresse des enfants. Paris : L'Harmattan.

Beguery, J. (2002). Une esthétique contemporaine de l'album de jeunesse, de grands petits livres. Paris :

L'Harmattan.

Bergson, H. (2009). La pensée et le mouvant. Paris : Presses universitaires de France. 
Blomgren, H. (2019). Beauty bubbles, subtle meetings, and frames for play: Aesthetic processes in Danish kindergartens. International Journal of Education \& the Arts, 20(1). https://doi.org/10.18113/ P8ijea20n1

Brogowski,L. (2010). Editer l'art : le livre d'artiste et l'histoire du livre. Chatou : Edition de la Transparence.

Brogowski, L ; Moeglin-Delcroix, A. (2010). Du concept non élargi du livre et du concept élargi de l'art dans le livre d'artiste. Actes du colloque, Les dimensions sociales du livre d'artiste. Le livre d'artiste : quels projets pour l'art? (p.9-13). Dijon : Les presses du réel.

Brougère, G. (1995). Jeu et éducation. Paris : L'Harmattan.

Chirouter, E. (2015). L'enfant, la littérature et la philosophie. Paris : L'Harmattan.

Dewey, J. (1922). L'école et l'enfant. Delachaux et Niestlé.

Dewey, J. (1934/2014). L'art comme expérience (J.-P. Cometti et coll., trad.). Paris : Gallimard.

Dewey, J. (1975). Démocratie et Education. Paris : Armand Colin.

Dewey, J. (2018). Démocratie et éducation (1916) et Expérience et Education (1938). Paris : Armand Colin.

Dupeyrat, J. (2012). Les livres d'artistes entre pratiques alternatives à l'exposition et pratiques

d'expositions alternatives. [Thèse de doctorat en esthétique, Université de Rennes 2, Rennes, France]. https://tel.archives-ouvertes.fr/tel-00772314/document.

Eco, U. (1965). L'œuvre ouverte. Paris : Editions du Seuil

Jørgensen, D. (2018). The philosophy of imagination. T. Zittun \& V. Glaveanu (Eds.), Handbook of imagination and culture. 19-45.

Kerlan, A. (2004) L'art pour éduquer ? La tentation esthétique. Laval : Presses de l'Université Laval.

Bordeaux M-C., Kerlan, A. (aout 2016). L'évaluation des " effets » de l'éducation artistique et culturelle Étude méthodologique et épistémologique. Rapport final.

Merle, L-A. (2017). Le livre d'artiste pour enfants : une approche esthétique et socioculturelle de l'enfance. [Thèse de doctorat en sciences de l'éducation. Université Lumière Lyon 2, Lyon, France]. https:// tel.archives-ouvertes.fr/tel-00772314/document.

Moeglin-Delcroix, A. (2012). Esthétique du livre d'artiste, 1960-1980. Marseille : Le Mot et le Reste.

Nussbaum, M. (2011). Les émotions démocratiques. Comment former le citoyen du XXIe siècle? Paris :

climats. Flammarion.

Petit, M. (2014). Lire le monde : expérience de transmission culturelle aujourd'hui. Paris : Belin

Prince, N. (2010). La littérature de jeunesse en question(s). Rennes : Collection "interférences", presses universitaires de Rennes.

Prince, N, (2010). La littérature de jeunesse, pour une théorie littéraire. Paris : Armand Colin.

Ricoeur, P. (2015). Soi-même comme un autre. Paris : Points.

Ricoeur, P. (1983). Temps et récit. Tome I. Paris : Editions du Seuil.

Ricoeur, P. (1986). Du texte à l'action. Essais d'herméneutique. Paris : Editions du Seuil.

Ruby, Ch. (2002). La figure du spectateur. Paris : Armand Colin/Recherches. 
Thomasset, A. (2005). L'imagination dans la pensée de Paul Ricœur fonction poétique du langage et transformation du sujet. Etudes théologiques et religieuses. 80 (4), 525-541.

Turin, J. (2008). Ces livres qui font grandir les enfants. Paris : Didier Jeunesse.

Turin, J. (2009). Savoir d'où l'on vient, pour savoir où l'on va ! La revue 303 arts, recherches, créations, Les livres d'enfants, 17-28.

Zask, J. (2007). Pratiques artistiques et conduites démocratiques, Noesis, (11), 103-115.

Zask, J. (2014). Black Mountain college: Art, démocratie, utopie. Rennes : PUR.

\section{ABSTRACTS}

The artists'Book can be a way for learning democratic emotions (Nussbaum,2011). This learning is aimed at cultural training enabling the child to become an aware actor in society. It is postulated that a cultural practice combining the artistic book and workshops led by artists would promote this learning. Co-creation and experience will facilitate the development of democratic emotions.

Through the prism of the philosophy of education but also of aesthetics (Kerlan, 2004; Blomgren, 2019), an analysis of a device (Merle,2017) implemented over a period of two years with children aged 4 to 5 in a kindergarten will be proposed. Two kinds of data collected were retained: verbatim of the children's comments and the observation of theirs actions at the start and at the end of the experiment. We observe the transition from a practice of imitation to a work of cocreation with the artist's book. The workshop becomes a place where the body engages, allows itself to have an experience (Dewey,1934) and a communicative act (Gadamer, 1996; Habermas, 1981). After a presentation of the device and of the first artist's book used during the experiment, the conditions and characteristics of this particular object will be identified allowing to qualify it as revealing of form of co-education and co-creation in learning democratic emotions.

Le livre artistique peut être un outil pour l'apprentissage des émotions démocratiques (Nussbaum, 2011). Cet apprentissage vise une formation culturelle permettant à l'enfant de devenir un acteur conscient de la société. On postule qu'une pratique culturelle alliant le livre artistique et les ateliers menés par des artistes favoriserait cet apprentissage.

Par un regard au prisme de la philosophie de l'éducation mais aussi de l'esthétique (Kerlan, 2004 ; Blomgren, 2019), une analyse d'un dispositif (Merle, 2017) mis en œuvre sur une période de deux ans auprès d'enfants de moyenne et grande section de maternelle est proposée. Deux types de données récoltées ont été retenues : le verbatim des propos des enfants et l'observation de leurs postures en début et fin d'expérimentation. On observe le passage d'une pratique dite d'imitation à un travail de co-création avec le livre et/ou l'artiste. L'atelier devient un lieu où le corps s'engage, s'autorise à vivre une expérience (Dewey, 1934), laissant s'exprimer «l'agir communicatif » de Gadamer (1996) ou "communicationnel» de Habermas (1981). Après une brève présentation $\mathrm{du}$ dispositif et des premiers livres artistiques utilisés lors de l'expérimentation, il conviendra de voir les conditions et caractéristiques de cet objet artistique permettant de le qualifier de révélateur d'une forme de co-création dans l'apprentissage des émotions démocratiques.

\section{INDEX}

Mots-clés: apprentissage des émotions, livre d'artiste, co-création, expérience 
AUTHOR

LILA MERLE

Université Lyon 2 